Article

\title{
Lipocalin-2 Inhibits Osteosarcoma Cell Metastasis by Suppressing MET Expression via the MEK-ERK Pathway
}

\author{
Ko-Hsiu Lu ${ }^{1,2,+}+^{D}$, Jia-Sin Yang ${ }^{3,4,+}$, Yi-Hsien Hsieh ${ }^{3,4} \mathbb{D}$, Hsiao-Ju Chu ${ }^{3,4}$, Chia-Hsuan Chou ${ }^{3,4}$, \\ Eric Wun-Hao Lu ${ }^{5}$, Chiao-Wen Lin ${ }^{6,7, *}$ and Shun-Fa Yang ${ }^{3,4, *(D)}$
}

1 Department of Orthopedics, Chung Shan Medical University Hospital, Taichung 402, Taiwan; cshy307@csh.org.tw

2 School of Medicine, Chung Shan Medical University, Taichung 402, Taiwan

3 Institute of Medicine, Chung Shan Medical University, Taichung 402, Taiwan; gazn_sheep@yahoo.com.tw (J.-S.Y.); hyhsien@csmu.edu.tw (Y.-H.H.); pig191919@seed.net.tw (H.-J.C.); wishwing1109@gmail.com (C.-H.C.)

4 Department of Medical Research, Chung Shan Medical University Hospital, Taichung 402, Taiwan

5 American School in Taichung, Taichung 406, Taiwan; 21ericl@ast.tc.edu.tw

6 Institute of Oral Sciences, Chung Shan Medical University, Taichung 402, Taiwan

7 Department of Dentistry, Chung Shan Medical University Hospital, Taichung 402, Taiwan

* Correspondence: cwlin@csmu.edu.tw (C.-W.L.); ysf@csmu.edu.tw (S.-F.Y.); Tel.: +886-4-24739595-34253 (S.-F.Y.)

+ These authors contributed equally to the work.

check for updates

Citation: Lu, K.-H.; Yang, J.-S.; Hsieh, Y.-H.; Chu, H.-J.; Chou, C.-H.; Lu, E.W.-H.; Lin, C.-W.; Yang, S.-F. Lipocalin-2 Inhibits Osteosarcoma Cell Metastasis by Suppressing MET Expression via the MEK-ERK Pathway. Cancers 2021, 13, 3181. https://doi.org/10.3390/

cancers13133181

Academic Editor: Levi J. Beverly

Received: 3 June 2021

Accepted: 22 June 2021

Published: 25 June 2021

Publisher's Note: MDPI stays neutral with regard to jurisdictional claims in published maps and institutional affiliations.

Copyright: (C) 2021 by the authors Licensee MDPI, Basel, Switzerland. This article is an open access article distributed under the terms and conditions of the Creative Commons Attribution (CC BY) license (https:/ / creativecommons.org/licenses/by/ $4.0 /)$.
Simple Summary: Higher neutrophil-derived cytokine lipocalin-2 (LCN2) expression possesses a versatile role in a myriad of cancers, but little is known about the role of LCN2 on osteosarcoma metastasis. In this study, we demonstrated that higher LCN2 inhibited cellular motility, migration, and invasion of osteosarcoma cells. Moreover, the phosphorylation of extracellular signal-regulated kinase (ERK) 1/2 was decreased by LCN2 knockdown. Conclusively, LCN2 inhibits osteosarcoma cell metastasis by suppressing MET via the mitogen-activated protein kinases/ERK kinase (MEK)ERK pathway.

Abstract: Higher neutrophil-derived cytokine lipocalin-2 (LCN2) expression possesses a versatile role in a myriad of cancers, but little is known about the role of LCN2 on osteosarcoma metastasis. In this study, we demonstrated that higher LCN2 inhibited cellular motility, migration, and invasion of osteosarcoma cells. Moreover, using RNA sequencing technology, we found that LCN2 repressed MET gene expression in U2OS cells. Manipulation of LCN2 levels influenced the migratory potential of osteosarcoma cells as cellular migration was enhanced by transfecting with vectors containing a constitutively active LCN2 cDNA and recombinant human LCN2. Moreover, the phosphorylation of mitogen-activated protein kinases/extracellular signal-regulated kinase (ERK) kinase (MEK) 1/2 and ERK 1/2 was decreased by LCN2 knockdown. Furthermore, the use of ERK inhibitor (U0126) and activator ( $\mathrm{tBHQ}$ ) confirmed that the pharmaceutic inhibition of MEK-ERK augmented the LCN2mediated MET suppression and migration of U2OS and HOS cells. Conclusively, LCN2 inhibits osteosarcoma cell metastasis by suppressing MET via the MEK-ERK pathway.

Keywords: LCN2; metastasis; osteosarcoma; MET

\section{Introduction}

Cancer, associated with high mortality and disability rates, is one of the world's largest health problems. Osteosarcoma, mainly arising from the metaphysis of long bones, is the most primary bone malignancy with a peak of incidence at 10-15 years and the second incidence peak in older adulthood [1,2]. Because the high rate of metastasis is a defining feature of osteosarcoma, its metastasis rate is responsible for the great majority of treatment failures and high mortality rates. Surgical en bloc resection or amputation of the diseased 
limb to achieve a complete radical excision had been the most common form of treatment for most osteosarcomas prior to metastasis [3], whereas $80 \%$ of patients had pulmonary metastasis (perhaps undetectable) at the time of presentation [4,5]. Recently, the combination of surgery and chemotherapy for osteosarcoma has increased long-term survival chances to approximately $68 \%$ through limb salvaging surgeries based on radiological staging, surgical techniques, and new chemotherapy protocols $[3,6]$. However, potent metastatic transfer to the lungs is still the main target of development of new therapeutic targets for anti-metastasis of osteosarcoma.

Cancer metastasis, the growth of primary tumor cells in a distant organ, includes cell migration, invasion, intravasation, traveling via circulation, extravasation, and eventual arrest at distant secondary sites [7]. In the invasion-metastasis cascade, mitogen-activated protein kinases (MAPKs), a family of serine/threonine kinases including extracellular signal-regulated kinase (ERK) 1/2, c-Jun N-terminal kinase (JNK) 1/2, and p38, are activated by MAPK/ERK kinase (MEK, MAPKK) to participate in the pathway progression of cell metastases [8]. The MET gene, first identified as an oncogene in the 1980s [9], encodes a transmembrane glycoprotein which possesses tyrosine kinase activity and its primary single chain precursor protein is post-translationally cleaved to produce the alpha and beta subunits to form the mature receptor for the hepatocyte growth factor (HGF) [10]. The MET receptor tyrosine kinase (c-Met) is highly expressed in more than $80 \%$ of osteosarcoma samples and its expression is correlated with high metastatic potential and a poor prognosis [11,12]. In osteosarcoma cells and preclinical models, the inhibition of c-Met effectively suppresses the metastatic phenotype [13].

Lipocalin 2 (LCN2), a prominent member of the lipocalin superfamily in human activated neutrophils, is originally identified as a $25-\mathrm{kDa}$ secreted glycoprotein based on its covalent binding to the matrix metalloproteinase (MMP)-9 [14]. Higher LCN2 expression in cancerous cells compared with non-cancerous cells [15] and a pro-neoplastic role for LCN2 and related mechanisms have been demonstrated [16]. Since MMP-9 can degrade the extracellular matrix and basement membranes [17], LCN2 protects MMP-9 from degradation, thus increasing MMP-9 activity [18] to contribute to tumor progression and metastasis through formation of an LCN2/MMP complex [19]. Silencing of LCN2 represses invasion through a reduction in LCN2/MMP-9 complex formation in cholangiocarcinoma cells [20], which is linked to reduced survival of patients with cholangiocarcinoma of higher LCN2 expression [21]. However, LCN2 negatively modulates epithelial-mesenchymal transition (EMT) in hepatocellular carcinoma cells through the epidermal growth factor (EGF) or transforming growth factor (TGF)- $\beta 1 / \mathrm{LCN} 2 /$ Twist1 pathway [22]. In colon cancer cells, LCN2 may function as a metastasis suppressor [23]. Therefore, controversies related to the diverse effects of LCN2 remain, and especially in osteosarcoma are still unknown. Here, we tested the potential of LCN2 on human osteosarcoma metastasis and also investigated its underlying mechanisms.

\section{Materials and Methods}

\subsection{Materials}

Cell culture materials included Dulbecco's modified Eagle medium (DMEM) and minimum essential medium (MEM) purchased from Gibco-BRL (Gaithersburg, MD, USA), and fetal bovine serum (FBS) purchased from Hyclone Laboratories, Inc. (South Logan, UT, USA). Propidium iodide (PI) and antibodies specific for p38, phosphorylated p38, and $\beta$-actin were obtained from BD Biosciences (San Jose, CA, USA). Additionally, antibodies specific for MET, MEK1/2, ERK1/2 and JNK1/2, and phosphorylated MEK1/2, ERK1/2, and JNK1/ 2 were purchased from Cell Signaling Technology (Danvers, MA, USA). Antibodies specific for LCN2 were purchased from R\&D Systems (Minneapolis, MN, USA). Unless otherwise specified, all chemicals used in this study were purchased from Sigma-Aldrich (St. Louis, MO, USA). 


\subsection{Cell Culture}

The human osteosarcoma HOS (13-yr-old female), MG-63 (14-yr-old male), and U2OS (15-yr-old female) cell lines were obtained from the Food Industry Research and Development Institute (Hsinchu, Taiwan), and the Saos-2 (11-yr-old Caucasian female) cell line was obtained from American Type Culture Collection (Manassas, VA, USA). They were supplemented with $10 \%$ FBS, $1 \%$ penicillin/streptomycin, and $5 \mathrm{~mL}$ of glutamine and cultured in DMEM and Eagle's MEM media, respectively, and maintained at $37^{\circ} \mathrm{C}$ in the humidified atmosphere of a $5 \% \mathrm{CO}_{2}$ incubator as described elsewhere [24].

\subsection{Protein Extraction and Western Blot Analysis}

To investigate the molecular mechanism further, signaling pathways were detected by means of Western blot analysis. We plated $8 \times 10^{5} /$ dish HOS cells with or without overexpression of LCN2 and $6 \times 10^{5}$ /dish U2OS cells with or without knockdown of LCN2 on 6-cm cell culture dishes for $16 \mathrm{~h}$. The total cell lysates of HOS and U2OS cells were prepared as described in previous studies [25], and Western blot analysis was performed using specific primary antibodies against LCN2, MEK1/2, or 3 MAPKs (ERK1/2, JNK1/2, and p38) with the specific antibodies for unphosphorylated or phosphorylated forms of the corresponding MEK1/2, ERK1/2, JNK1/2, or p38. As previously described, blots were then incubated with a horseradish peroxidase goat anti-rabbit or anti-mouse IgG for $1 \mathrm{~h}$ and the intensity of each band was measured via densitometry.

\subsection{Transient Transfection}

Cell transfection was carried out using LipofectAMINE 2000 (Invitrogen, Life Technologies, Carlsbad, CA) according to the manufacturer's instructions. Vectors containing a constitutively active LCN2 cDNA ( $5 \mu \mathrm{g})$ and MET $(5 \mu \mathrm{g})$ were diluted in $200 \mu \mathrm{L}$ of MEM and then mixed with the transfection solution for $30 \mathrm{~min}$ as previously described [25]. Two days after transfection, the cells were used for the following experiment. Collected cells were processed for Western blot analysis, reverse transcription-polymerase chain reaction (RT-PCR), and cell migration assay.

\subsection{Small Interfering $R N A$ (siRNA)}

To silence LCN2 protein expression, siRNA-inhibiting human LCN2 (s8114) and negative-control siRNA (4390844) were purchased from Applied Biosystems Instruments (Foster City, CA, USA). For the experiment, $6 \times 10^{5} /$ dish U2OS cells were grown on $6 \mathrm{~cm}$ cell culture dishes overnight. Following the manufacturer's instructions, a total of $30 \mathrm{pM}$ of LCN2 siRNA was transfected into the cells using lipofectamine RNAiMAX reagent (Invitrogen, Carlsbad, CA, USA). The silencer negative control siRNA, a nonsense siRNA duplex, was used as a control [24,26]. Similarly, a total of $50 \mathrm{pM}$ of MET siRNA (s8700) was transfected into U2OS cells to silence MET protein expression as previously described.

\subsection{Microculture Tetrazolium Colorimetric (MTT) Assay}

For the cell viability experiment, we plated $8 \times 10^{4} /$ well HOS cells with or without overexpression of LCN2 and $8 \times 10^{4} /$ well U2OS cells with or without silencing of LCN2 in 24-well plates for 6 days, and a (3-(4,5-dimethylthiazol-2-yl)-2,5-diphenyltetrazolium bromide) (MTT) assay was performed to determine the cytotoxicity of LCN2 overexpression and knockdown. The media were removed and the HOS and U2OS cells were washed with phosphate-buffered saline. The media were subsequently changed and the cells were incubated with MTT $(0.5 \mathrm{mg} / \mathrm{mL})$ for $4 \mathrm{~h}$ as previously described [25].

\subsection{Reverse Transcription-Polymerase Chain Reaction (RT-PCR)}

For RT-PCR, we plated $8 \times 10^{5} /$ dish HOS cells and $6 \times 10^{5} /$ dish U2OS cells with or without silencing of LCN2 or MET, $4 \times 10^{5} /$ dish MG- 63 cells, and $4 \times 10^{5} /$ dish Saos-2 cells on 6-cm culture dishes for $24 \mathrm{~h}$. The RNA was entirely extracted using Total RNA Mini Kit (Geneaid, New Taipei City, Taiwan) and reverse transcribed into complementary 
DNA (cDNA) using a High Capacity cDNA Reverse Transcription Kit (Applied Biosystems, Foster City, CA, USA). Procedures of cDNA synthesis and the PCR amplification assay were performed as described in a previous study $[24,25]$. Specific primers were used for LCN2 and MET genes. For LCN2, the following forward (F) primers, and reverse (R) primers were used: F: 5'-TGATCCCAGCCCCACCT-3', R: 5'-CCACTTCCCCTGGAATTGGT-3'. For SYBR-MET, the following forward (F) primers, and reverse $(\mathrm{R})$ primers were used: $\mathrm{F}$ : 5'ATACggTCCTATggCTggTg3', R: 5'TTgAgAggTTCTTTCCACCAAgT3'.

\subsection{Flow Cytometry}

To estimate the proportion of HOS and U2OS cells in different phases of cell cycle affected by transfecting with pcDNA vector for overexpression of LCN2 and siRNA directly against the LCN2 expression, cellular DNA contents and apoptosis were measured by flow cytometry as described elsewhere [27]. Using propidium iodide staining, cell cycle analysis by DNA content measurement employed flow cytometry to distinguish cells in different phases of the cell cycle.

\subsection{Wound Healing Assay}

To determine whether LCN2 alters the motility of HOS and U2OS cells, we plated $8 \times 10^{5}$ / well HOS cells with or without LCN2 overexpression and $6 \times 10^{5} /$ well U2OS cells with or without LCN2 knockdown on 6-well plates for $48 \mathrm{~h}$ and wounded them by scratching with a pipette tip. Afterwards, the cells were incubated with DMEM containing $0.5 \%$ FBS for $0,4,8$, and $24 \mathrm{~h}$ for HOS cells and for $0,6,9$, and $12 \mathrm{~h}$ for U2OS cells. Following previous studies, the cells were photographed using a phase-contrast microscope $(\times 100)[26]$.

\subsection{Cell Migration Assay}

To examine the role of LCN2 on migration of U2OS and HOS cells in vitro, we employed a modified Boyden chamber without Matrigel coating. HOS cells with or without overexpression of LCN2 and U2OS cells with or without silencing of LCN2 were seeded into the upper section of the Boyden chamber (Neuro Probe, Cabin John, MD, USA) at densities of $2 \times 10^{5} / \mathrm{mL}$ for HOS cells and $3 \times 10^{5} / \mathrm{mL}$ for U2OS cells, and then incubated for $24 \mathrm{~h}$ at $37^{\circ} \mathrm{C}$. Subsequently, the cells of migration were counted under a light microscope [25].

\subsection{MET Promoter-Driven Luciferase Reporter Assay}

A density of $6 \times 10^{5} /$ dish U2OS cells with or without silencing of LCN2 were plated on 6-well plates for $24 \mathrm{~h}$. The pGL3-basic vector, $\mathrm{pGL3}$-control vector, and pGL3-MET promoter $(-926$ to +261$)$ were co-transfected with a $\beta$-galactosidase expression vector (pCH110) as previously described. After 24 and $48 \mathrm{~h}$ of transfection, cell lysates were harvested, and luciferase activity was determined using a luciferase assay kit. The value of the luciferase activity was normalized to transfection efficiency and monitored by $\beta$-galactosidase expression [28].

\subsection{Statistical Analysis}

Statistical calculations of the data were performed using Student's t-test for the differences between two independent samples and one-way analysis of variance (ANOVA) with post hoc Scheffe and Tukey's tests for more than two groups with unequal and equal sample sizes per group, respectively. Each experiment was performed in triplicate and three independent experiments were performed. A $p$-value $<0.05$ was considered statistically significant. 


\section{Results}

3.1. Inconsistent LCN2 Expressions in Human Osteosarcoma HOS, MG-63, Saos-2, and U2OS Cells

To assess expressions of LCN2 in human osteosarcoma HOS, MG-63, Saos-2, and U2OS cells, Western blot analysis and RT-PCR were utilized. Our results expectedly showed inconsistent LCN2 mRNA and protein expressions in different cell lines: lower LCN2 mRNA and protein expressions were observed in HOS, MG-63, and Saos-2 cells; and the highest expressions were observed in U2OS cells, which showed about 10 times more of them in HOS, MG-63, and Saos-2 cells in RT-PCR (Figure 1A,B). Accordingly, we chose HOS and U2OS cells in all subsequent experiments to investigate the role of LCN2 in mechanisms underlying modulation of osteosarcoma metastasis.

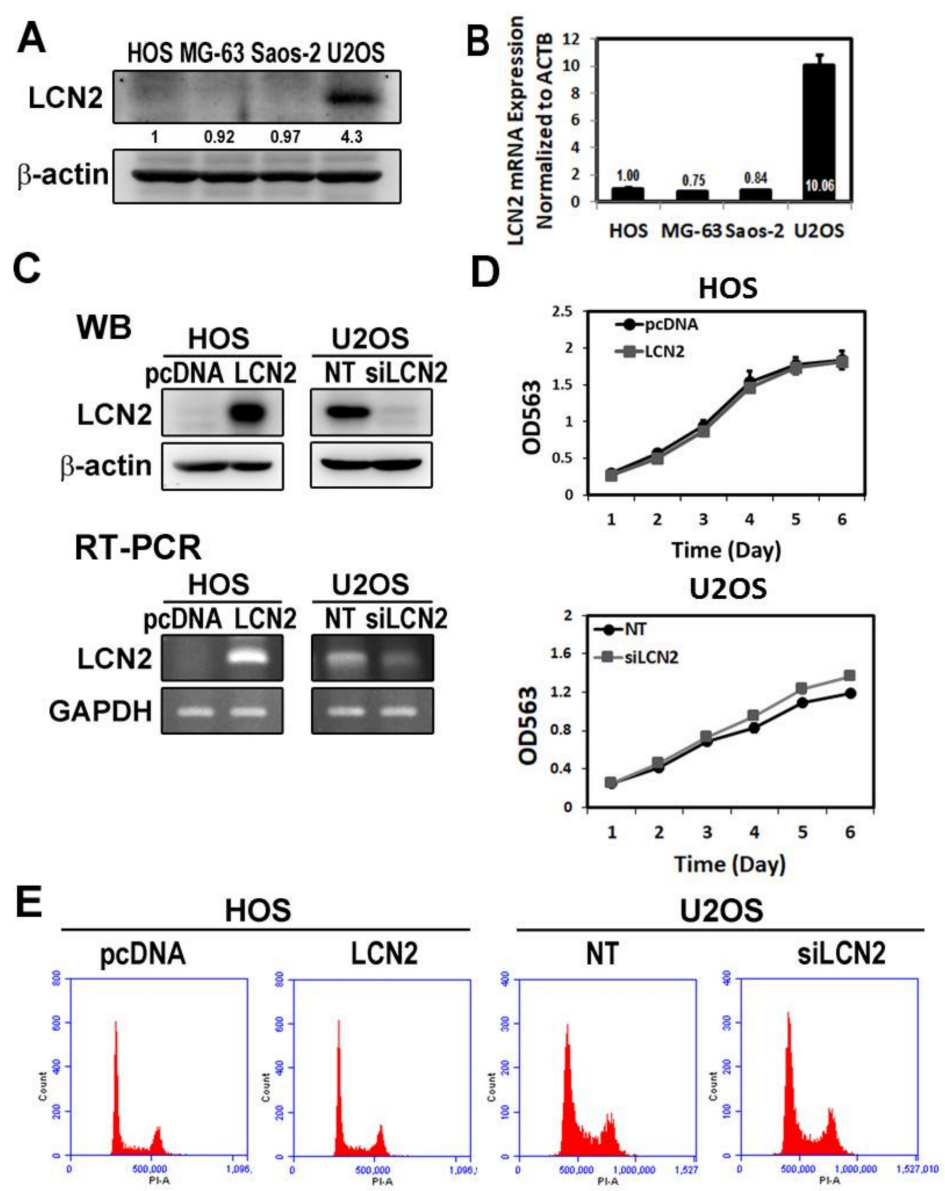

Figure 1. Effects of LCN2 on cell viability of human osteosarcoma cells. (A) Using Western blot analysis and (B) RT-PCR, the LCN2 protein and mRNA expressions in human osteosarcoma HOS, MG-63, Saos-2, and U2OS cells were detected, respectively. (C) Western blot analysis and RT-PCR for LCN2 protein and mRNA expressions of HOS cells after transfection with vectors containing a constitutively active LCN2 cDNA and U2OS cells after siRNA directly against the LCN2 expression were conducted. (D) Using an MTT assay, cell viability of HOS cells after transfection with vectors containing a constitutively active LCN2 cDNA and U2OS cells after siRNA directly against the LCN2 expression was detected up to 6 days and the effects are illustrated after quantitative analysis. (E) HOS cells after transfection with vectors containing a constitutively active LCN2, cDNA, and U2OS cells after siRNA directly against the LCN2 expression were subjected to flow cytometry after PI staining to analyze the cell cycle regulation. 
3.2. Motility and Migration Are Inhibited in HOS Cells by Overexpression of LCN2 but Promoted in U2OS Cells by Silencing of LCN2

To determine whether LCN2 influences cellular motility, invasion, and migration, transfection with pcDNA vector for overexpression of LCN2 for HOS cells and siRNA directly against the LCN2 expression for U2OS cells was employed. We confirmed overexpression and knockdown of LCN2 protein and mRNA levels through Western blotting and RT-PCR in HOS and U2OS cells, respectively (Figure 1C). To demonstrate their cytotoxicity, MTT assay and flow cytometry showed that overexpression of LCN2 in HOS cells and silencing of LCN2 in U2OS cells up to 6 days did not increase the incidence of apoptosis, as evidenced by the absence of significant changes in the cell cycle (Figure 1D,E).

To verify the anti-metastatic actions of LCN2, we employed wound healing and modified Boyden chamber migration assays using HOS cells with or without overexpression of LCN2 and U2OS cells with or without silencing of LCN2 to compare cellular motility and migration. Intriguingly, overexpression of LCN2 significantly repressed motility and migratory potential in HOS cells, whereas LCN2 knockdown significantly enhanced those potentials in U2OS cells (wound healing: HOS: $4 \mathrm{~h}: p<0.05,8 \mathrm{~h}: p<0.01,24 \mathrm{~h}$ : $p<0.01$; U2OS: 6 h: $p<0.001,9$ h: $p<0.001,12$ h: $p<0.001$; migration: HOS: $p<0.001$, U2OS: $p<0.001$ ) (Figure 2A-C). To further test the potential effect of LCN2 expression on human osteosarcoma metastasis, the recombinant LCN2 protein and culture media of LCN2 overexpression HOS cells were used. By using recombinant human LCN2 in HOS cells, we confirmed that LCN2 dose-dependently suppressed the migratory response in the modified Boyden chamber migration assay (F: 132,128, $p<0.001$ ) (Figure 2D). Culture media of LCN2 overexpression HOS cells significantly decreased the migratory potential of both HOS and U2OS cells, as expected ( $p<0.001$ and $p<0.001)$ (Figure 2E).

\subsection{LCN2 Inhibits MET mRNA and Protein Expressions in HOS and U2OS Cells}

To explore the underlying mechanisms of the anti-metastatic actions of LCN2 in osteosarcoma cells, we conducted a transcriptomic analysis of U2OS cells transfected with siRNA inhibiting human LCN2 for $24 \mathrm{~h}$ using RNA sequencing technology. During the RNA sequencing analysis in siLCN2 U2OS cells, the expression levels of two upregulated DEG candidates, MET and PTPMT1, and two downregulated DEG candidates, BLOC1S4 and TSPAN13, were verified. Among them, MET is the most significantly upregulated gene in LCN2-knockdown cells and there are some reports regarding MET and osteosarcoma metastasis [11-13]. Furthermore, only one report regards promotion of human osteosarcoma cells progression by enhancing TSPAN13 expression [29]. Therefore, we chose the MET gene as the target gene to investigate its anti-metastatic properties. To validate the RNA sequencing findings of MET, we conducted Western blot analysis and interestingly found that HOS cells with LCN2 overexpression decreased the MET expression, while U2OS cells with LCN2 knockdown increased the MET expression (Figure 3B). Culture media of LCN2 overexpression of HOS cells expectedly suppressed the MET expression in both U2OS and HOS cells (Figure 3C). In RT-PCR, HOS cells treated with an overexpression of LCN2 significantly increased the LCN2 mRNA expression; U2OS cells treated with LCN2 knockdown significantly decreased the LCN2 mRNA expression $(p<0.001$ and $p<0.001)$. Predictably, the MET mRNA expression was significantly decreased in HOS cells by overexpression of LCN2 but increased in U2OS cells by silencing of LCN2 $(p<0.001$ and $p<0.001$ ) (Figure 3D). In RT-PCR, both U2OS cells and HOS cells treated with culture media of LCN2 overexpression HOS cells significantly decreased the MET mRNA expression $(p<0.001$ and $p<0.001)$, and recombinant human LCN2 also significantly down-regulated the MET mRNA expression in HOS cells (F: 12,791, $p<0.01$ ) (Figure 3E). Collectively, the results implied that secreted glycoprotein LCN2 modulates expressions of the MET mRNA and protein. 

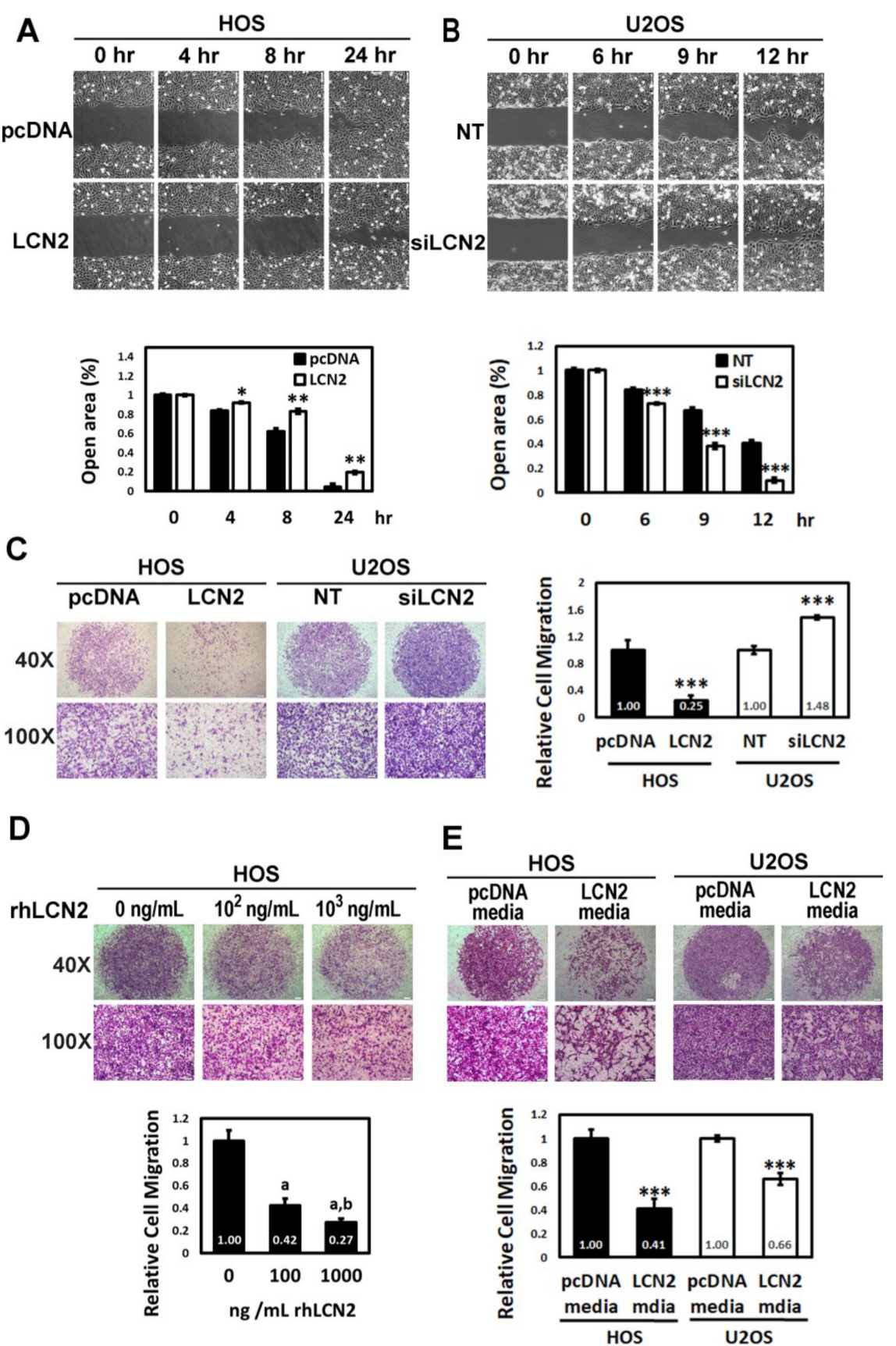

Figure 2. Effects of LCN2 on cell motility and migration of human osteosarcoma cells. $(\mathbf{A}, \mathbf{B})$ Effects of HOS cells after transfection with vectors containing constitutively active LCN2 cDNA and U2OS cells after siRNA directly against the LCN2 expression in wound healing assay were measured. ${ }^{*} p<0.05$, ** $p<0.01$, and ${ }^{* * *} p<0.001$ compared with the vehicle group. (C) Using a cell migration assay, migratory HOS cells after transfection with vectors containing a constitutively active LCN2 cDNA and U2OS cells after siRNA directly against the LCN2 expression were measured and subsequently subjected to quantitative analysis. ${ }^{* * *} p<0.001$ compared with the vehicle group. (D,E) Cell migration assays for HOS cells after treatment with various concentrations $(0,100$, and $1000 \mathrm{ng} / \mathrm{mL})$ of recombinant human LCN2 and both HOS and U2OS cells after treatment with culture media of LCN2 overexpression HOS cells were measured and subsequently subjected to quantitative analysis. F: $132.128, p<0.001$. ${ }^{a}$ Significantly different, $p<0.05$, when compared with control. ${ }^{b}$ Significantly different, $p<0.05$, when compared with $100 \mathrm{ng} / \mathrm{mL}$. ${ }^{* *} p<0.001$ compared with the vehicle group. 
A

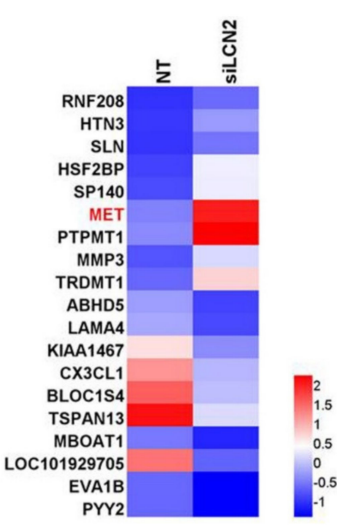

B

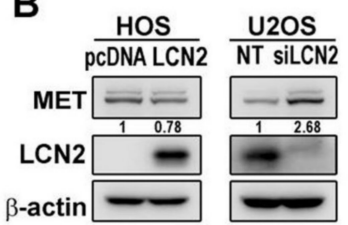

C

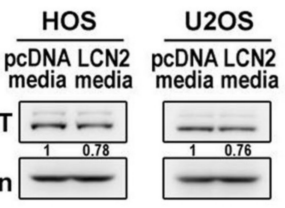

D
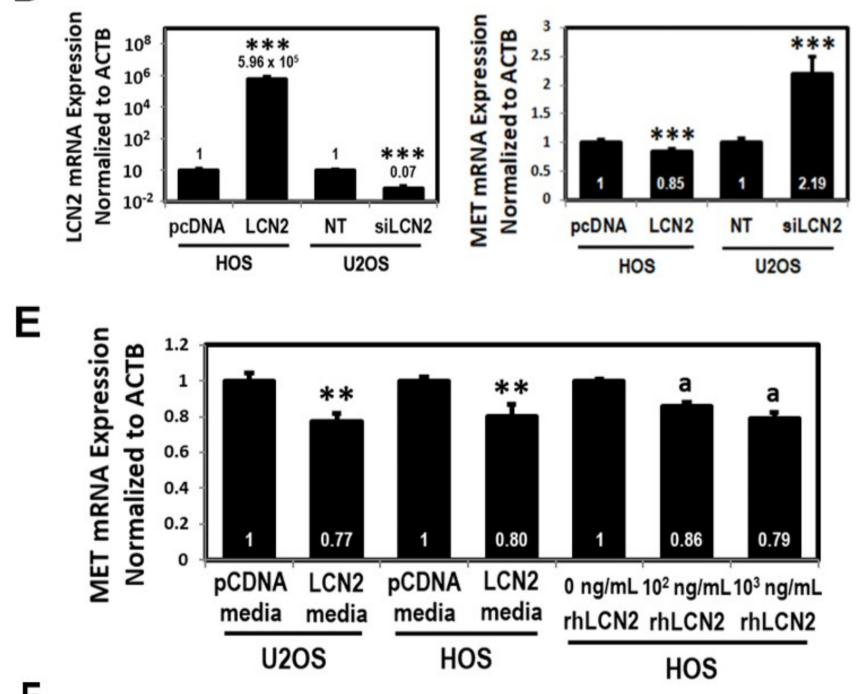

$\mathbf{F}$
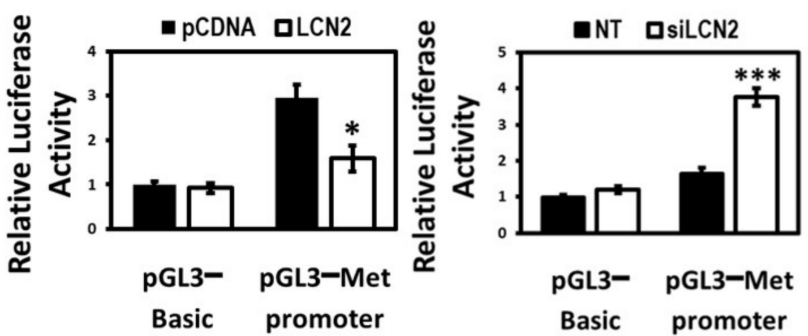

Figure 3. RNA sequencing for siLCN2 U2OS cells and effects of LCN2 on MET in HOS and U2OS cells. (A) Heat map of the hierarchical clustering of 19 differentially expressed genes identified in U2OS cells after siRNA directly against the LCN2 expression for $24 \mathrm{~h}$. (B) Western blot analyses for the LCN2 and MET expressions in HOS cells after transfection with vectors containing a constitutively active LCN2 CDNA and U2OS cells after siRNA directly against the LCN2 expression were conducted. (C) Western blot analyses for the MET expression in both U2OS and HOS cells treated with culture media of LCN2 overexpression HOS cells were conducted. (D) RT-PCR for LCN2 and MET mRNA expressions in HOS cells after transfection with vectors containing a constitutively active LCN2 cDNA and U2OS cells after siRNA directly against the LCN2 expression were measured and subsequently subjected to quantitative analysis. ${ }^{* * *} p<0.001$ compared with the vehicle group. (E) RT-PCR for the MET mRNA expression in both U2OS and HOS cells treated with culture media of LCN2 overexpression HOS cells and HOS cells treated with various concentrations $(0,100$, and $1000 \mathrm{ng} / \mathrm{mL})$ of recombinant human LCN2 were measured and subsequently subjected to quantitative analysis. ${ }^{* *} p<0.01$ compared with the vehicle group. F: $12,791, p<0.01$. ${ }^{a}$ Significantly different, $p<0.05$, when compared with control. (F) HOS cells were transfected with pcDNA vector for overexpression of LCN2 and U2OS cells were transfected with siRNA inhibiting human LCN2 for $24 \mathrm{~h}$ and then subjected to luciferase assay to analyze the promoter activity of MET. ${ }^{*} p<0.05,{ }^{* * *} p<0.001$ compared with the vehicle group. 
We further identify whether LCN2 inhibits the promoter activity of MET to regulate the gene expression in U2OS cells. The luciferase reporter assay revealed that overexpression of LCN2 significantly decreased the promoter activity of MET in HOS cells while silence of LCN2 enhanced the promoter activity of MET in U2OS cells (Figure 3F), indicating the LCN2-induced inhibition of the mRNA of MET in U2OS cells at least partially at a transcriptional level.

\subsection{MET Promotes U2OS and HOS Cells Migration}

To validate whether MET is the downstream molecule of LCN2 and influences toward cell migration, U2OS cells transfected with vectors containing a constitutively active MET and siRNA directly against the MET expression were employed. Unsurprisingly, Western blot analysis showed that both overexpression and silencing of MET could not affect the LCN2 expression in U2OS cells (Figure 4A). In RT-PCR, MET knocked-down U2OS cells significantly decreased the MET mRNA expression and MET overexpression U2OS cells significantly increased the MET mRNA expression $(p<0.001$ and $p<0.001)$, whereas both the MET knockdown and MET overexpression U2OS cells had no effect on LCN2 mRNA expression $(p=0.79$ and $p=0.71)$, indicating that MET is not an upstream signal of LCN2. Moreover, the modified Boyden chamber migration assay showed that MET overexpression in U2OS cells significantly increased cellular migration and reversed the effect of MET silencing, significantly decreasing U2OS cellular migration $(p<0.001$ and $p<0.001$ ), (Figure 4B).

Transfection with vectors containing a constitutively active MET cDNA significantly increased migration of HOS cells without overexpression of LCN2 and reversed the decrease in migration of HOS cells with LCN2 overexpression, but had no effect on the LCN2 expression in HOS cells with or without LCN2 overexpression (F: 82,987, $p<0.001$ ). In U2OS cells with or without knockdown of LCN2, transfection with siRNA directly against the MET expression significantly decreased migration of U2OS cells without knockdown of LCN2 and reversed the increase in migration in LCN2 knockdown U2OS cells, without affecting the LCN2 expression (F: 150,955, $p<0.001$ ) (Figure 4C-E). Therefore, the results confirmed that MET is downstream signing of LCN2 to modulate migration of HOS and U2OS cells.

\subsection{LCN2 Inhibits the MEK-ERK Pathway to Repress Migration of U2OS and HOS Cells}

Since MEK and MAPKs pathways may be downstream signaling of LCN2, Western blot analysis was employed to further investigate its molecular mechanisms. In the analysis, MEK1/2 and MAPKs pathways were detected in HOS cells with or without overexpression of LCN2 and U2OS cells with or without knockdown of LCN2. As a result, LCN2 overexpression HOS cells decreased phosphorylation of MEK1/2 and ERK but LCN2 knockdown U2OS cells increased phosphorylation of MEK1/2 and ERK, indicating that LCN2 inhibits phosphorylation of MEK1/2 and ERK (Figure 5A). Additionally, increased phosphorylation of JNK1/2 in LCN2 overexpression HOS cells, decreased phosphorylation of JNK1/2 in LCN2 knockdown U2OS cells, and no obvious influence on p38 in both HOS and U2OS cells, including its phosphorylation, were observed. Based on these findings, the MEK-ERK cascade is suppressed by LCN2. 
A

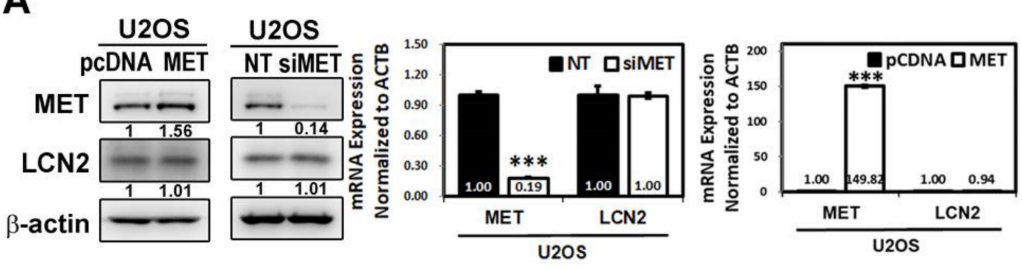

B
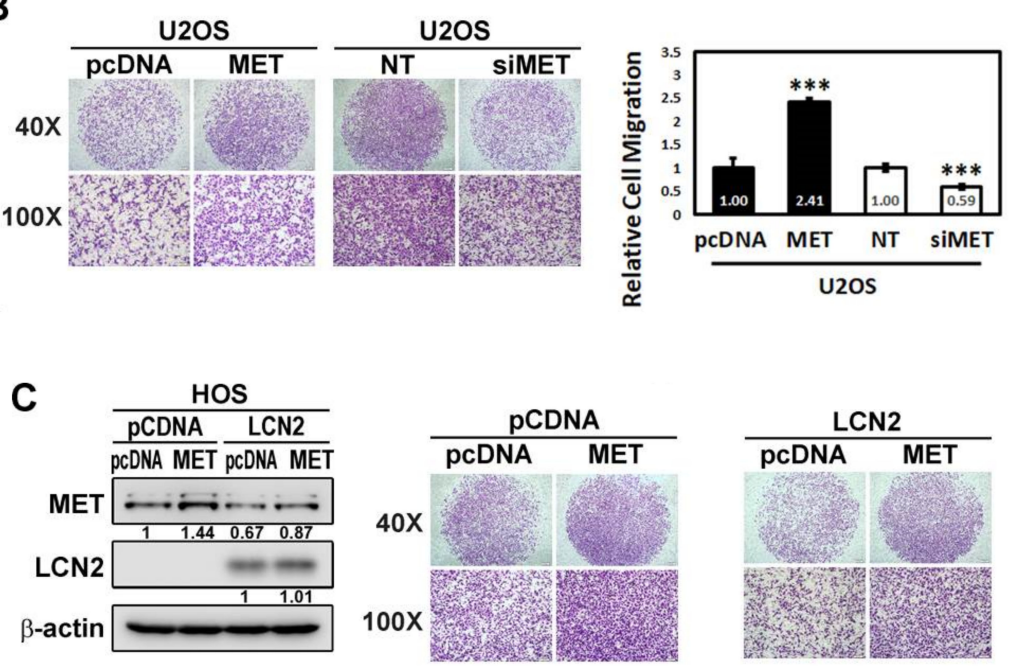

D
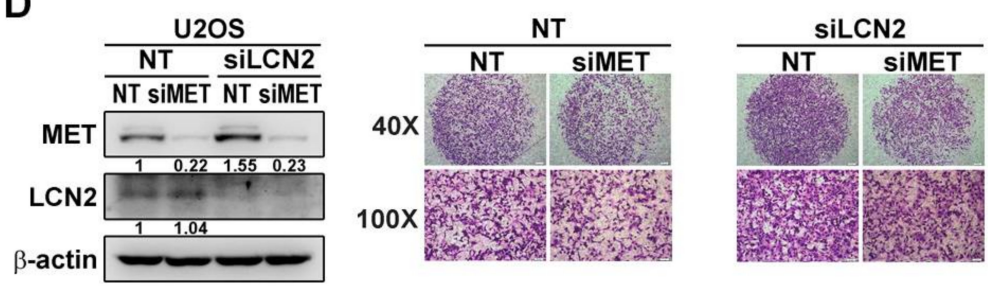

E
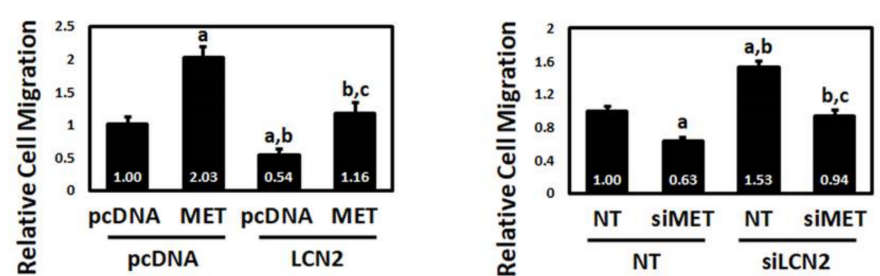

Figure 4. Effects of MET on U2OS and HOS cells migration. (A) Western blot analyses for MET and LCN2 proteins in U2OS cells after transfection with vectors containing a constitutively active MET cDNA and siRNA directly against the MET expression were measured. RT-PCR for MET and LCN2 mRNA expressions in U2OS cells after siRNA directly against the MET expression and U2OS cells after transfection with vectors containing a constitutively active MET cDNA were measured and subsequently subjected to quantitative analysis. ${ }^{* *} p<0.001$ compared with the vehicle group. (B) Cell migration assays for U2OS cells after transfection with vectors containing a constitutively active MET cDNA and siRNA directly against the MET expression were measured and subsequently subjected to quantitative analysis. ${ }^{* * *} p<0.001$ compared with the vehicle group. (C-E) Western blot analyses and cell migration assays for HOS cells with or without overexpression of LCN2, transfected with vectors containing a constitutively active MET cDNA, and U2OS cells with or without knockdown of LCN2, siRNA directly against the MET expression, were measured, respectively, and migratory cells

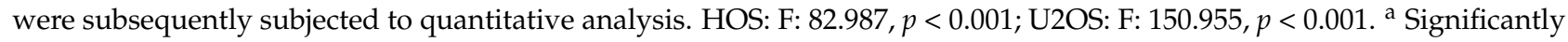
different, $p<0.05$, when compared with HOS cells without overexpression of LCN2 and MET, or U2OS cells without silencing of LCN2 and MET. ${ }^{b}$ Significantly different, $p<0.05$, when compared with HOS cells without overexpression of

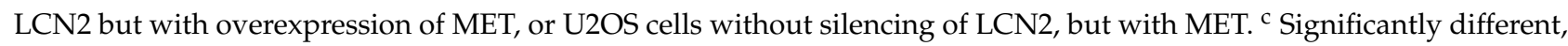
$p<0.05$, when compared with HOS cells with overexpression of LCN2 but without overexpression of MET, or U2OS cells with silencing of LCN2, but without silencing of MET. 


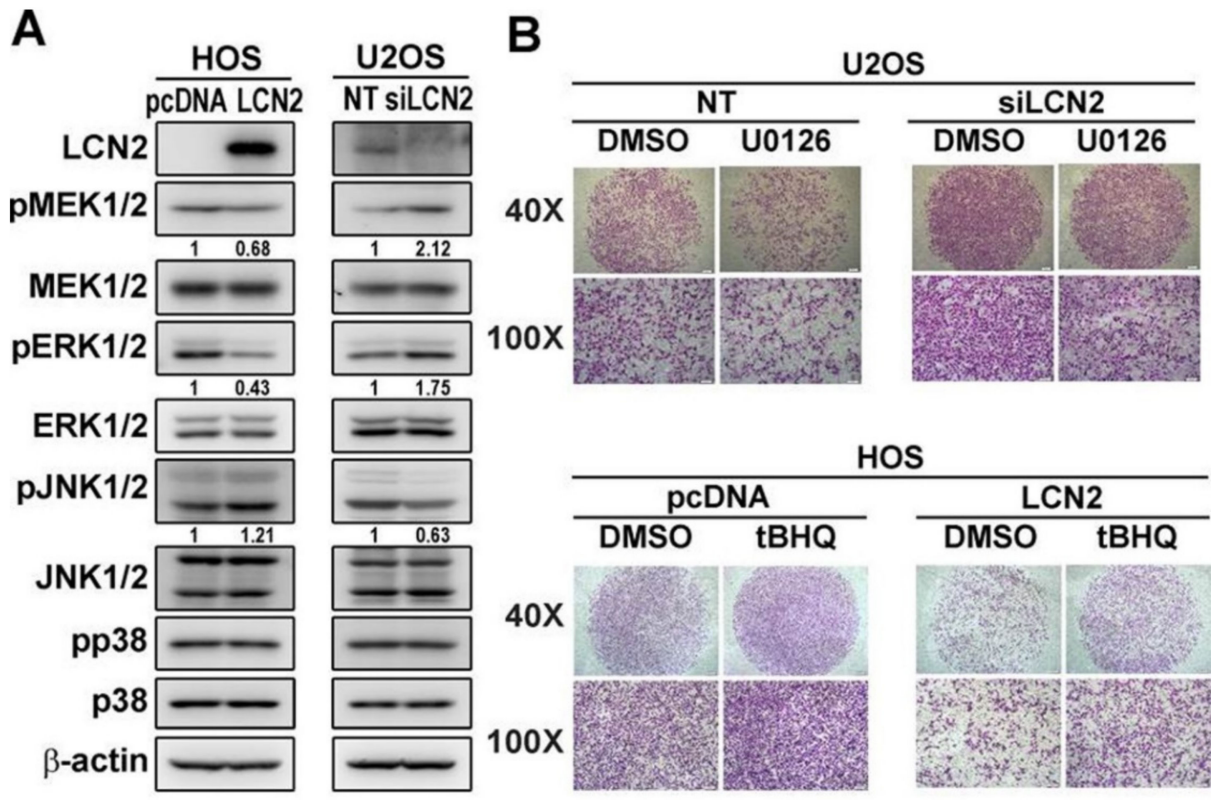

C
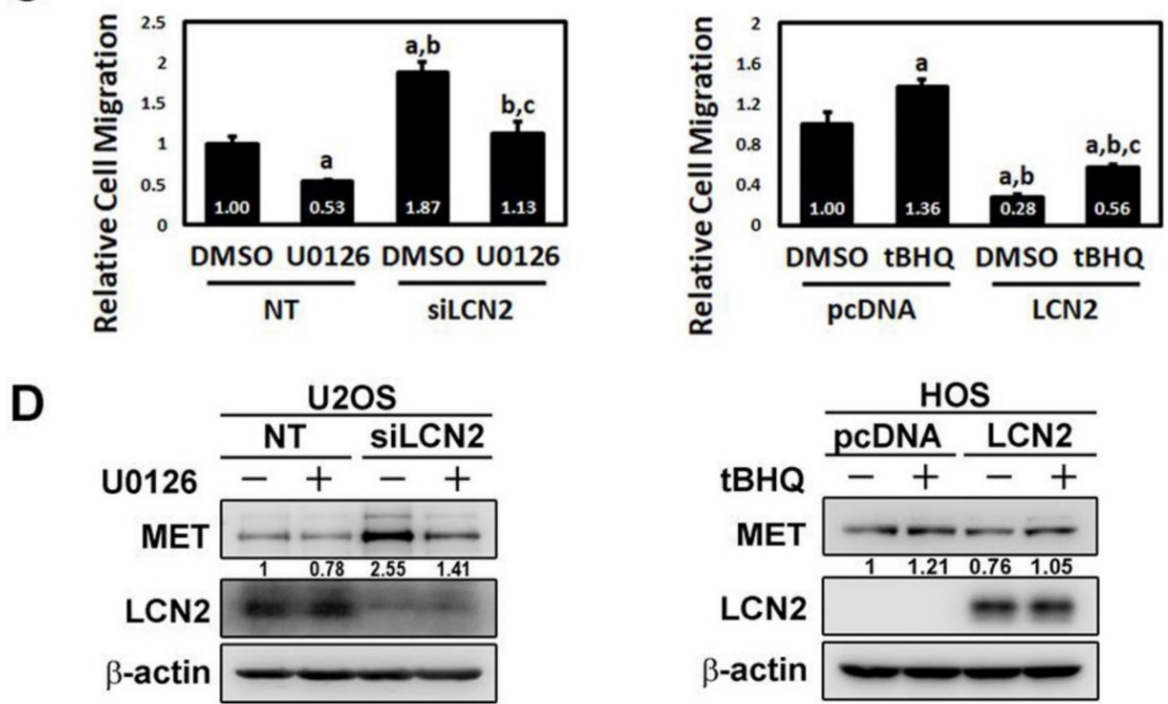

Figure 5. Effects of LCN2 on MEK and MAPKs to modulate MET and migration in U2OS and HOS cells. (A) Western blot analyses for HOS cells with or without overexpression of LCN2 and U2OS cells with or without knockdown of LCN2 to determine MEK and MAPKs, including their phosphorylation, were conducted, respectively. (B,C) Cell migration assays for effects of the inhibitor of ERK (U0126) on U2OS cells with or without silencing of LCN2 and the activator of ERK (tBHQ) on HOS cells with or without overexpression of LCN2 were measured and subsequently subjected to quantitative analysis. U2OS: F: 110,894, $p<0.001$; HOS: F: 168,737, $p<0.001$. ${ }^{a}$ Significantly different, $p<0.05$, when compared with U2OS cells without silencing of LCN2 and without U0126, or HOS cells without overexpression of LCN2 and without tBHQ. b Significantly different, $p<0.05$, when compared with U2OS cells without silencing of LCN2 but with U0126, or HOS cells without overexpression of LCN2 but with tBHQ. ${ }^{c}$ Significantly different, $p<0.05$, when compared with U2OS cells with silencing of LCN2 but without U0126, or HOS cells with overexpression of LCN2 but without tBHQ. (D) Western blot analysis for U2OS cells with or without silencing of LCN2 and HOS cells with or without overexpression of LCN2 to determine effects of U0126 and tBHQ on MET and LCN2 expressions were conducted. 
To further identify whether the inhibition of MEK-ERK phosphorylation by LCN2 interferes with the suppression of MET and cellular migration, we used the inhibitor of ERK (U0126) in U2OS cells with or without LCN2 knockdown and the activator of ERK (tBHQ) in HOS cells with or without LCN2 overexpression in Western blot analysis. As shown in Figure 5B,C, migratory potential of U2OS cells was activated by silencing of LCN2 (F: 110,894, $p<0.001$ ). Conversely, migratory potential of HOS cells was repressed by overexpression of LCN2 (F: 168,737, $p<0.001$ ). Interestingly, the inhibitor of ERK (U0126) significantly decreased the migratory response of U2OS cells with or without silencing of LCN2 $(p<0.05$ and $p<0.05)$ and also significantly reduced the increase in migration in LCN2 knockdown U2OS cells $(p<0.05)$. Migratory potential of HOS cells with or without overexpression of LCN2 was significantly increased by the activator of ERK (tBHQ) (both $p<0.05$ ). Expectedly, the activator of ERK (tBHQ) significantly reversed the decrease in migration of LCN2 overexpression HOS cells $(p<0.05)$. Taken together, these results provided better defining of the MEK-ERK signaling pathway which acts as downstream of LCN2.

\subsection{LCN2-Inhibited Migration through Suppression of MET via the MEK-ERK Pathway in U2OS and HOS Cells}

To further confirm whether the MEK-ERK pathway is localized at upstream of MET, Western blotting was subsequently performed. While the MET expression was predictably activated by silencing of LCN2 and reduced by the inhibitor of ERK (U0126) in U2OS cells with or without LCN2 knockdown, their LCN2 expressions could not be influenced by U0126. In HOS cells with or without overexpression of LCN2, the MET expression was repressed by LCN2 overexpression and enhanced by the activator of ERK (tBHQ), whereas tBHQ had no effect on LCN2 expressions (Figure 5D). Overall, these findings indicated that the MEK-ERK pathway plays a critical upstream role of MET in HOS and U2OS cells.

\section{Discussion}

As expected, we demonstrated inconsistent expressions of the cell-derived immunerelated protein LCN2 in human osteosarcoma HOS, MG-63, Saos-2, and U2OS cells. Because the opposite effects of $\mathrm{LCN}$ on metastatic potential in two human colon cancer cell lines are very intriguing [23], we further explored the manipulation of LCN2 without cytotoxicity reducing cellular motility, invasiveness, and migratory potential in low and high LCN2 expressions of HOS and U2OS cells, respectively. Using RNA sequencing technology and the pathway enrichment analysis, the upregulated protein of MET was intriguingly observed after silencing of LCN2 in U2OS cells. To validate the RNA sequencing finding related to MET, we further confirmed that overexpression of LCN2 and recombinant human LCN2 in HOS cells, and culture media of LCN2 overexpression HOS cells in HOS and U2OS cells repressed mRNA and protein expressions of MET, whereas MET mRNA and protein expressions in U2OS cells were activated by silencing of LCN2. Nevertheless, both transfection with vectors containing a constitutively active MET cDNA and siRNA directly against the MET expression could not affect the LCN2 expression in HOS and U2OS cells. Migration of HOS cells with or without overexpression of LCN2 was induced by MET overexpression, whereas migration of U2OS cells with or without silencing of LCN2 was repressed by MET knockdown. Through a further analysis of upstream pathways of MET in LCN2-inhibited migration, we discovered that overexpression of LCN2 decreased phosphorylation of MEK1/2 and ERK1/2 in HOS cells, while silencing of LCN2 increased phosphorylation of MEK1/2 and ERK1/2 in U2OS cells. Notably, the inhibitor of ERK (U0126) attenuated the MET expression and migration in U2OS cells with or without LCN2 knockdown and the activator of ERK (tBHQ) promoted the MET expression and migration in HOS cells with or without LCN2 overexpression. These findings implied that LCN2's anti-metastatic actions in human osteosarcoma HOS and U2OS cells resulted from attenuation of the MET expression and activity through the MEK-ERK pathway rather than JNK and p38 signaling. 
In addition to some inflammation and pathological conditions [30,31], the versatile cytokine LCN2 has gained attention as a potential biomarker and a modulator of some human cancers, including breast [14], thyroid [32], colon [33], and pancreatic cancers [34]. In metastasis, LCN2 is initially identified as a promotor to induce EMT in breast cancer cells to enhance tumor invasion [35]. In prostate cancer cells, LCN2 plays an important role in facilitating cell migration and invasion of prostate cancer by inducing EMT through the ERK/SLUG axis [36], and the CXCL1-LCN2 axis triggers a cascade amplification event of Src signaling, EMT, and migration, leading to promotion of cancer metastasis [37]. However, disruption of the LCN2 gene in mice suppresses primary mammary tumor formation, whereas it does not decrease lung metastasis [38]. Thus, controversies over LCN2's function remain in different cancer types.

Even in the same genetic origin, increasing LCN2 expression in poorly metastatic human colon cancer (KM12C) cells generates more invasive abilities while LCN2 short hairpin RNA has the opposite effect; nevertheless, over-expressing LCN2 in the highly metastatic human colon cancer (KM12SM) cell line greatly reduces its invasive behavior in vitro and in vivo [23]. Furthermore, LCN2 promotes EMT through Rac1, one of Ras homolog gene family (Rho) small guanosine triphosphate hydrolases (GTPases) and an integral regulator of EMT induction, to increase KM12C cell motility and invasion, indicating that LCN2 may be a target for therapeutic intervention in colorectal cancer cell metastasis [39]. Based on metastatic potential varied between the two cell lines used, the discrepancy between reports may be due to a difference in cell line specificity or to the fact that it is critical in advancing metastatic potential. Therefore, the role of LCN2 in anti-metastasis is remarkably divergent not only among the different cancer types but also between various cell lines in the same cancer. Using manipulation of LCN2 in low LCN2 expression of HOS cells and high LCN2 expression of U2OS cells, we confirmed LCN2 contributing to the anti-metastatic property in human osteosarcoma cells regardless of originally high or low expressions.

Generally, MET is thought to play a central role in signaling pathways to control cell proliferation, survival, and migration, in response to binding by its ligand HGF during developmental morphogenesis and in multiple cancer types [9,40]. In particular, expressions of MET/HGF receptor in osteosarcoma with and without bone metastasis were noted in 25 and $90 \%$ of cases, respectively [41]. However, we are intrigued that interactive crosstalk exists between MET and other members of the EGF receptor (EGFR) family, including human EGFR (HER)2 and HER3, and MET can be regulated through intermediary signaling pathways because of different requirements for MAPK signaling depending on different cell types, lines, and contexts [11,42]. For example, EGF-activated MET phosphorylation occurs through ERK and p38 pathways and compensation works via parallel MAPK cascades in mouse myeloid 32D cells [43]. A more complicated relationship presents in EGFR-MET crosstalk in non-small cell lung carcinoma cells of varying metastatic potential and EGFR modulates MET at multiple levels to enhance cellular migration and invasion, as demonstrated by our findings of a new tumor-specific crosstalk for the LCN2's anti-metastatic action by attenuating MET through the MEK-ERK pathway in human osteosarcoma cells. To further investigate the inhibitory level of LCN2 on MET, we performed the luciferase reporter assay and observed that LCN2 knockdown U2OS cells significantly enhanced the MET promoter activity. Consequently, this indicates that downregulation of LCN2 on the MET expression in U2OS cells occurs at least partially at a transcriptional level.

Hence, we confirmed the novel discovery that illustrated the anti-metastatic property of LCNS involving the MEK-ERK pathway and downstream MET in human osteosarcoma. These data suggest a need for future research on anti-metastasis of LCN2 for osteosarcoma and render the molecular basis for better understanding of LCN2 as a novel anti-metastatic target for research of osteosarcoma in the future. Indeed, how it attenuates metastasis may be clear now and a pertinent concern is actually determining which molecules are involved in the MEK-ERK pathway when LCN2 inhibits the migratory ability of HOS and U2OS 
cells. Nevertheless, the limitations of this study include the lack of an in vivo study and the absence of information on how to manipulate LCN2 herein to translate it to clinical use; therefore, further studies are required to validate whether the anti-metastatic actions in vivo occurs as those in vitro.

\section{Conclusions}

Conclusively, human osteosarcoma HOS and U2OS cell-derived LCN2 contributes to inhibition of cellular migration through the MEK-ERK signaling pathway to downstream MET, and these findings imply an identified biomarker of LCN2 on anti-metastasis of osteosarcoma. The promotion of LCN2 expression to repress motility, invasion, and migration could be a potential target for anti-metastasis of osteosarcoma. This new discovery illustrates the involvement of the MEK-ERK pathway and downstream MET on migration of osteosarcoma, which in turn illustrates the pertinence of further studying LCN2 related to anti-metastasis for human osteosarcoma.

Author Contributions: Conceptualization, K.-H.L., J.-S.Y., C.-W.L., and S.-F.Y.; methodology, J.-S.Y., Y.-H.H., H.-J.C., C.-H.C., and E.W.-H.L.; writing—original draft preparation, K.-H.L., J.-S.Y., C.-W.L., and S.-F.Y.; writing-review and editing, K.-H.L., C.-W.L., and S.-F.Y. All authors have read and agreed to the published version of the manuscript.

Funding: This work was supported by grants from the Ministry of Science and Technology, Taiwan (MOST-105-2314-B-040-003-MY3.) This research was also supported by Chung Shan Medical University Hospital, Taiwan (CSH-2021-D-011).

Institutional Review Board Statement: Not applicable.

Informed Consent Statement: Not applicable.

Data Availability Statement: The data presented in this study are available on request from the corresponding author.

Conflicts of Interest: The authors declare no conflict of interest.

\section{References}

1. Mirabello, L.; Troisi, R.J.; Savage, S.A. Osteosarcoma incidence and survival rates from 1973 to 2004: Data from the surveillance, epidemiology, and end results program. Cancer 2009, 115, 1531-1543. [CrossRef]

2. Picci, P.; Mercuri, M.; Ferrari, S.; Alberghini, M.; Briccoli, A.; Ferrari, C.; Pignotti, E.; Bacci, G. Survival in high-grade osteosarcoma: Improvement over 21 years at a single institution. Ann. Oncol. 2010, 21, 1366-1373. [CrossRef] [PubMed]

3. Ottaviani, G.; Jaffe, N. The epidemiology of osteosarcoma. Cancer Treat. Res. 2009, 152, 3-13.

4. Dorfman, H.D. Osteosarcoma. In Bone Tumors; Dorfman, H.D., Czerniak, B., Eds.; Mosby: St Louis, MO, USA, 1998 ; pp. $128-252$.

5. Weis, L. Common malignment bone tumors: Osteosarcoma. In Surgery for Bone and Soft-Tissue Tumors; Simon, M.A., Springfield, D., Eds.; Lippincott-Raven: Philadelphia, PA, USA, 1997; pp. 265-274.

6. Oertel, S.; Blattmann, C.; Rieken, S.; Jensen, A.; Combs, S.E.; Huber, P.E.; Bischof, M.; Kulozik, A.; Debus, J.; Schulz-Ertner, D. Radiotherapy in the treatment of primary osteosarcoma-A single center experience. Tumori J. 2010, 96, 582-588. [CrossRef]

7. Lu, K.H.; Lin, C.W.; Su, S.C.; Reiter, R.J.; Yang, S.F. New insights into anti-metastatic signaling pathways of melatonin in skeletomuscular sarcoma of childhood and adolescence. Cancer Metastasis Rev. 2020, accepted.

8. Chan-Hui, P.Y.; Weaver, R. Human mitogen-activated protein kinase kinase kinase mediates the stress-induced activation of mitogen-activated protein kinase cascades. Biochem. J. 1998, 336 Pt 3, 599-609. [CrossRef]

9. Cooper, C.S.; Park, M.; Blair, D.G.; Tainsky, M.A.; Huebner, K.; Croce, C.M.; Vande Woude, G.F. Molecular cloning of a new transforming gene from a chemically transformed human cell line. Nature 1984, 311, 29-33. [CrossRef]

10. Cooper, C.S. The met oncogene: From detection by transfection to transmembrane receptor for hepatocyte growth factor. Oncogene 1992, 7, 3-7. [PubMed]

11. Maroun, C.R.; Rowlands, T. The met receptor tyrosine kinase: A key player in oncogenesis and drug resistance. Pharmacol. Ther. 2014, 142, 316-338. [CrossRef] [PubMed]

12. Scagliotti, G.V.; Novello, S.; von Pawel, J. The emerging role of met/hgf inhibitors in oncology. Cancer Treat. Rev. 2013, 39, 793-801. [CrossRef]

13. MacEwen, E.G.; Kutzke, J.; Carew, J.; Pastor, J.; Schmidt, J.A.; Tsan, R.; Thamm, D.H.; Radinsky, R. C-met tyrosine kinase receptor expression and function in human and canine osteosarcoma cells. Clin. Exp. Metastasis 2003, 20, 421-430. [CrossRef]

14. Yang, J.; Bielenberg, D.R.; Rodig, S.J.; Doiron, R.; Clifton, M.C.; Kung, A.L.; Strong, R.K.; Zurakowski, D.; Moses, M.A. Lipocalin 2 promotes breast cancer progression. Proc. Natl. Acad. Sci. USA 2009, 106, 3913-3918. [CrossRef] 
15. Candido, S.; Maestro, R.; Polesel, J.; Catania, A.; Maira, F.; Signorelli, S.S.; McCubrey, J.A.; Libra, M. Roles of neutrophil gelatinase-associated lipocalin (ngal) in human cancer. Oncotarget 2014, 5, 1576-1594. [CrossRef] [PubMed]

16. Chakraborty, S.; Kaur, S.; Guha, S.; Batra, S.K. The multifaceted roles of neutrophil gelatinase associated lipocalin (ngal) in inflammation and cancer. Biochim. Biophys. Acta 2012, 1826, 129-169. [CrossRef]

17. Chang, P.Y.; Hsieh, M.J.; Hsieh, Y.S.; Chen, P.N.; Yang, J.S.; Lo, F.C.; Yang, S.F.; Lu, K.H. Tricetin inhibits human osteosarcoma cells metastasis by transcriptionally repressing mmp-9 via p38 and akt pathways. Environ. Toxicol. 2017, 32, 2032-2040. [CrossRef] [PubMed]

18. Yan, L.; Borregaard, N.; Kjeldsen, L.; Moses, M.A. The high molecular weight urinary matrix metalloproteinase (mmp) activity is a complex of gelatinase $\mathrm{b} / \mathrm{mmp}-9$ and neutrophil gelatinase-associated lipocalin (ngal). Modulation of mmp-9 activity by ngal. $J$. Biol. Chem. 2001, 276, 37258-37265. [CrossRef] [PubMed]

19. Candido, S.; Abrams, S.L.; Steelman, L.S.; Lertpiriyapong, K.; Fitzgerald, T.L.; Martelli, A.M.; Cocco, L.; Montalto, G.; Cervello, M.; Polesel, J.; et al. Roles of ngal and mmp-9 in the tumor microenvironment and sensitivity to targeted therapy. Biochim Biophys Acta 2016, 1863, 438-448. [CrossRef] [PubMed]

20. Nuntagowat, C.; Leelawat, K.; Tohtong, R. Ngal knockdown by sirna in human cholangiocarcinoma cells suppressed invasion by reducing ngal/mmp-9 complex formation. Clin. Exp. Metastasis 2010, 27, 295-305. [CrossRef]

21. Chiang, K.C.; Yeh, T.S.; Wu, R.C.; Pang, J.S.; Cheng, C.T.; Wang, S.Y.; Juang, H.H.; Yeh, C.N. Lipocalin 2 (lcn2) is a promising target for cholangiocarcinoma treatment and bile lcn2 level is a potential cholangiocarcinoma diagnostic marker. Sci. Rep. 2016, 6, 36138. [CrossRef]

22. Wang, Y.P.; Yu, G.R.; Lee, M.J.; Lee, S.Y.; Chu, I.S.; Leem, S.H.; Kim, D.G. Lipocalin-2 negatively modulates the epithelial-tomesenchymal transition in hepatocellular carcinoma through the epidermal growth factor (tgf-beta1)/lcn2/twist1 pathway. Hepatology 2013, 58, 1349-1361. [CrossRef]

23. Lee, H.J.; Lee, E.K.; Lee, K.J.; Hong, S.W.; Yoon, Y.; Kim, J.S. Ectopic expression of neutrophil gelatinase-associated lipocalin suppresses the invasion and liver metastasis of colon cancer cells. Int. J. Cancer 2006, 118, 2490-2497. [CrossRef] [PubMed]

24. Cheng, H.L.; Lin, C.W.; Yang, J.S.; Hsieh, M.J.; Yang, S.F.; Lu, K.H. Zoledronate blocks geranylgeranylation not farnesylation to suppress human osteosarcoma u2os cells metastasis by emt via rho a activation and fak-inhibited jnk and p38 pathways. Oncotarget 2016, 7, 9742-9758. [CrossRef] [PubMed]

25. Hsieh, Y.S.; Chu, S.C.; Yang, S.F.; Chen, P.N.; Liu, Y.C.; Lu, K.H. Silibinin suppresses human osteosarcoma mg-63 cell invasion by inhibiting the erk-dependent c-jun/ap-1 induction of mmp-2. Carcinogenesis 2007, 28, 977-987. [CrossRef]

26. Lu, K.H.; Su, S.C.; Lin, C.W.; Hsieh, Y.H.; Lin, Y.C.; Chien, M.H.; Reiter, R.J.; Yang, S.F. Melatonin attenuates osteosarcoma cell invasion by suppression of c-c motif chemokine ligand 24 through inhibition of the c-jun n-terminal kinase pathway. J. Pineal Res. 2018, 65, e12507. [CrossRef] [PubMed]

27. Lu, K.H.; Lue, K.H.; Chou, M.C.; Chung, J.G. Paclitaxel induces apoptosis via caspase-3 activation in human osteogenic sarcoma cells (u-2 os). J. Orthop. Res. 2005, 23, 988-994. [CrossRef] [PubMed]

28. Lu, K.H.; Yang, H.W.; Su, C.W.; Lue, K.H.; Yang, S.F.; Hsieh, Y.S. Phyllanthus urinaria suppresses human osteosarcoma cell invasion and migration by transcriptionally inhibiting u-pa via erk and akt signaling pathways. Food Chem. Toxicol. 2013, 52, 193-199. [CrossRef]

29. Jaiswal, R.K.; Kumar, P.; Kumar, M.; Yadava, P.K. Htert promotes tumor progression by enhancing tspan13 expression in osteosarcoma cells. Mol. Carcinog. 2018, 57, 1038-1054. [CrossRef]

30. Moschen, A.R.; Adolph, T.E.; Gerner, R.R.; Wieser, V.; Tilg, H. Lipocalin-2: A master mediator of intestinal and metabolic inflammation. Trends Endocrinol. Metab. 2017, 28, 388-397. [CrossRef]

31. Viau, A.; El Karoui, K.; Laouari, D.; Burtin, M.; Nguyen, C.; Mori, K.; Pillebout, E.; Berger, T.; Mak, T.W.; Knebelmann, B.; et al. Lipocalin 2 is essential for chronic kidney disease progression in mice and humans. J. Clin. Investig. 2010, 120, 4065-4076. [CrossRef]

32. Iannetti, A.; Pacifico, F.; Acquaviva, R.; Lavorgna, A.; Crescenzi, E.; Vascotto, C.; Tell, G.; Salzano, A.M.; Scaloni, A.; Vuttariello, E.; et al. The neutrophil gelatinase-associated lipocalin (ngal), a nf-kappab-regulated gene, is a survival factor for thyroid neoplastic cells. Proc. Natl. Acad. Sci. USA 2008, 105, 14058-14063. [CrossRef]

33. Birkenkamp-Demtroder, K.; Christensen, L.L.; Olesen, S.H.; Frederiksen, C.M.; Laiho, P.; Aaltonen, L.A.; Laurberg, S.; Sorensen, F.B.; Hagemann, R.; Ørntoft, T.F. Gene expression in colorectal cancer. Cancer Res. 2002, 62, 4352-4363. [PubMed]

34. Tong, Z.; Kunnumakkara, A.B.; Wang, H.; Matsuo, Y.; Diagaradjane, P.; Harikumar, K.B.; Ramachandran, V.; Sung, B.; Chakraborty, A.; Bresalier, R.S.; et al. Neutrophil gelatinase-associated lipocalin: A novel suppressor of invasion and angiogenesis in pancreatic cancer. Cancer Res. 2008, 68, 6100-6108. [CrossRef] [PubMed]

35. Yang, J.; Moses, M.A. Lipocalin 2: A multifaceted modulator of human cancer. Cell Cycle 2009, 8, 2347-2352. [CrossRef]

36. Ding, G.; Fang, J.; Tong, S.; Qu, L.; Jiang, H.; Ding, Q.; Liu, J. Over-expression of lipocalin 2 promotes cell migration and invasion through activating erk signaling to increase slug expression in prostate cancer. Prostate 2015, 75, 957-968. [CrossRef]

37. Lu, Y.; Dong, B.; Xu, F.; Xu, Y.; Pan, J.; Song, J.; Zhang, J.; Huang, Y.; Xue, W. Cxcl1-lcn2 paracrine axis promotes progression of prostate cancer via the src activation and epithelial-mesenchymal transition. Cell Commun. Signal. 2019, 17, 118. [CrossRef] [PubMed]

38. Berger, T.; Cheung, C.C.; Elia, A.J.; Mak, T.W. Disruption of the lcn2 gene in mice suppresses primary mammary tumor formation but does not decrease lung metastasis. Proc. Natl. Acad. Sci. USA 2010, 107, 2995-3000. [CrossRef] 
39. Hu, L.; Hittelman, W.; Lu, T.; Ji, P.; Arlinghaus, R.; Shmulevich, I.; Hamilton, S.R.; Zhang, W. Ngal decreases e-cadherin-mediated cell-cell adhesion and increases cell motility and invasion through rac1 in colon carcinoma cells. Lab. Investig. 2009, 89, 531-548. [CrossRef] [PubMed]

40. Trusolino, L.; Bertotti, A.; Comoglio, P.M. Met signalling: Principles and functions in development, organ regeneration and cancer. Nat. Rev. Mol. Cell Biol. 2010, 11, 834-848. [CrossRef]

41. Arihiro, K.; Inai, K. Expression of $\mathrm{cd} 31$, met/hepatocyte growth factor receptor and bone morphogenetic protein in bone metastasis of osteosarcoma. Pathol. Int. 2001, 51, 100-106. [CrossRef]

42. Lai, A.Z.; Abella, J.V.; Park, M. Crosstalk in met receptor oncogenesis. Trends Cell Biol. 2009, 19, 542-551. [CrossRef] [PubMed]

43. Breindel, J.L.; Haskins, J.W.; Cowell, E.P.; Zhao, M.; Nguyen, D.X.; Stern, D.F. Egf receptor activates met through mapk to enhance non-small cell lung carcinoma invasion and brain metastasis. Cancer Res. 2013, 73, 5053-5065. [CrossRef] [PubMed] 